\title{
Delaying/Reducing the Risk of Clinical Tumour Progression after Primary Curative Procedures
}

\author{
M. Wirth \\ Department of Urology, Technical University of Dresden, Germany
}

\section{Key Words}

Prostate cancer - Antiandrogen - Bicalutamide • Immediate therapy · Adjuvant therapy

\begin{abstract}
The advent of prostate-specific antigen (PSA) testing and increased patient awareness has led to patients being diagnosed with prostate cancer at an earlier stage and a younger age than previously. Adjuvant hormonal therapy to radiotherapy or prostatectomy has been shown to reduce the risk of tumour progression, and in some studies survival benefits have been demonstrated. The nonsteroidal antiandrogen bicalutamide ('Casodex') has undergone extensive evaluation and is currently undergoing clinical trials as immediate therapy, either alone or as adjuvant to treatment of curative intent in patients with localized or locally advanced disease. Data from the first analysis of one of the studies in the Early Prostate Cancer (EPC) programme involving 3,603 patients have shown that, after a median follow-up of 2.6 years, the risk of prostate cancer progression was significantly reduced (by $43 \%$ ) in patients receiving bicalutamide $150 \mathrm{mg}$ compared with those receiving standard care alone (HR 0.57; 95\% $\mathrm{Cl} 0.48,0.69 ; \mathrm{p} \ll 0.0001)$. The risk of PSA progression was also significantly reduced (by $63 \%$ ). At this stage the sur-
\end{abstract}

'Casodex' and 'Zoladex' are trade marks of the AstraZeneca group of companies

\section{KARGER}

Fax +41613061234

E-Mail karger@karger.ch

www. karger.com
(C) 2001 S. Karger AG, Basel

0302-2838/01/0408-0017\$17.50/0

Accessible online at:

www. karger.com/journals/eur vival data are still immature. Side effects of bicalutamide were mostly gynaecomastia and breast pain, which is consistent with its pharmacology. Overall withdrawal rates were similar in the bicalutamide $150 \mathrm{mg}$ and standard care alone groups. In the bicalutamide $150 \mathrm{mg}$ group, withdrawals were mainly due to side effects, whereas in the group receiving standard care alone, withdrawals were mainly due to disease progression. The programme is ongoing, and survival data are awaited.

Copyright @ 2001 S. Karger AG, Basel

\section{Introduction}

Prostate cancer is one of the leading causes of mortality in men, with the highest incidence of new cancer diagnosis [1]. The incidence of prostate cancer has increased due to improved awareness and diagnosis, with improved testing for prostate-specific antigen (PSA) levels enabling the disease to be detected at an earlier stage (clinically localized and locally advanced disease). Prostate cancer patients have increased longevity compared with patients with other types of cancer, such as breast or lung, and life expectancy can be more than 15 years [2]. Therefore, tolerability of long-term therapy must be considered. Many patients are sexually active, and so current therapies, which often inhibit sexual function, are not always desirable. In finding new treatment options, therapy must not only delay disease progression and prolong survival but must also provide a good quality of life [3].

Prof. M. Wirth

Universitätsklinikum Carl Gustav Carus

Klinik und Poliklinik für Urologie, Technische Universität Dresden

Fetscher Strasse 74, D-01307 Dresden (Germany)

Tel. +49 351458 4447, Fax +49 351458 4333, E-Mail wirth-m@rcs1.urz.tu-dresden.de 
Fig. 1. Probability of overall survival over a 10 -year period following treatment with radiotherapy plus goserelin compared with radiotherapy alone [11] (reproduced with kind permission from the Massachusetts Medical Society).

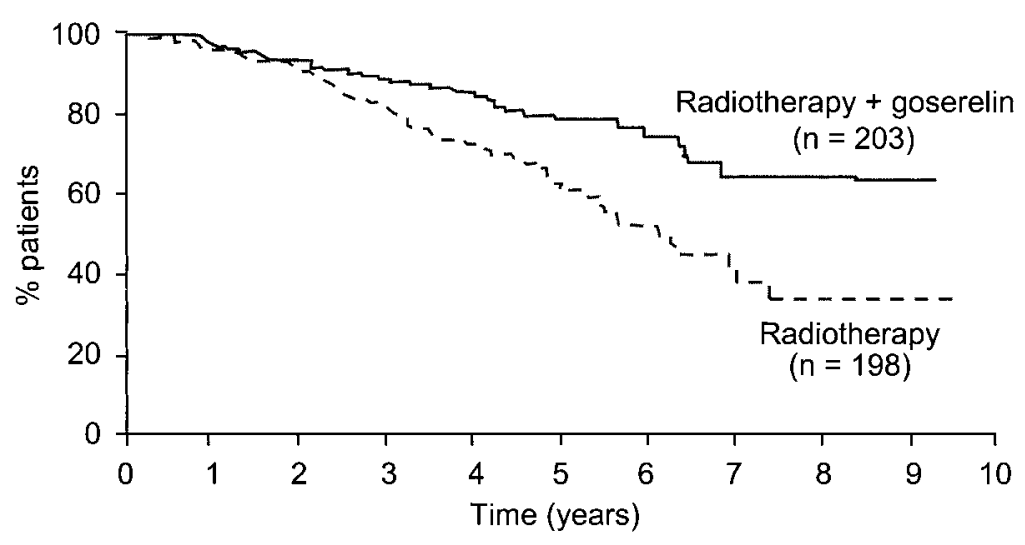

HR $0.50 ; 95 \% \mathrm{Cl} 0.33,0.76 ; \mathrm{p}=0.001$

\section{Treatment Options for Prostate Cancer}

One treatment for locally confined prostate cancer is radical prostatectomy. Although $84.7 \%$ of men with pT1$\mathrm{T} 2$ disease remain progression free 10 years after surgery, risk of disease progression is increased in patients with focal capsular penetration or established capsular penetration (67.7 vs. $58.4 \%$ remain progression-free 10 years after surgery, respectively) [4]. Radiotherapy is an alternative option for patients who are not able, or do not wish, to undergo surgery and for those patients with locally advanced disease. Clinical trials have shown that a patient with T1-T2, Nx disease (Gleason score 2-6) has a $28 \%$ risk of dying from prostate cancer 15 years after radiotherapy, but a patient with $\mathrm{T} 3, \mathrm{Nx}$ or $\mathrm{N}+$ disease (Gleason score $8-10$ ) has a $73 \%$ risk of dying from prostate cancer 15 years after therapy [5].

In order to minimize the risk of disease progression, and to minimize symptoms of prostate cancer for patients who have undergone primary therapy, adjuvant hormonal therapy can be administered. Luteinizing hormonereleasing hormone (LHRH) (agonists or antagonists) reduces androgen stimulation of tumour growth, as does orchiectomy. However, withdrawal of androgens leads to different side effects, such as impotence, osteoporotic fractures [6] and lowered haemoglobin levels [7], and also has negative psychological implications [8]. Therefore, there is interest in using non-steroidal antiandrogens, which do not have these side effects, in this setting.

Hormonal therapy can be initiated either immediately after primary treatment or at a later date, once PSA levels begin to rise or the patient begins to suffer from increased symptoms. The timing of hormonal therapy may depend upon the age of the patient, stage of disease and individual preference. Currently, the most appropriate timing of hormonal therapy is still a subject of much debate.

\section{Adjuvant Hormonal Therapy in the Radiotherapy Setting}

Administration of adjuvant therapy has the potential to improve survival. Isaacs $[9,10]$ demonstrated that a combination of chemotherapy and androgen ablation increased survival of rats when compared with the therapies given alone.

Clinical trials have since shown the beneficial effects of adjuvant hormonal therapy. Radiotherapy plus adjuvant hormonal therapy (goserelin ['Zoladex'] $3.6 \mathrm{mg}$ every 4 weeks for 3 years) administered to patients with locally advanced prostate cancer (stage T3-4, N0-2, M0) resulted in a significantly higher proportion of patients remaining disease free after 5 years compared with radiotherapy alone ( 85 vs. $48 \%$, respectively; $p<0.001$ ) [11]. Overall survival after 5 years was $79 \%$ in the adjuvant group compared with $62 \%$ in the group receiving radiotherapy alone ( $\mathrm{p}=0.001$; fig. 1$)$ [11].

The Radiation Therapy Oncology Group (RTOG) conducted a randomized Phase III trial of adjuvant goserelin (3.6 mg indefinitely or until disease progression) in patients receiving radiotherapy for stage $\mathrm{T} 1-\mathrm{T} 3$ prostate cancer [12]. After a median follow-up of 4.5 years there was a statistically significant improvement in progression-free survival in patients receiving adjuvant therapy compared with those receiving radiotherapy alone (84 vs. $71 \% ; \mathrm{p}<0.0001)$. The difference in 5 -year overall surviv- 
al was not statistically significant between the two groups, which may be due to all patients having early prostate cancer with an overall low risk of disease progression. However, in a planned subgroup analysis of patients with a poor prognosis with a Gleason score of 8-10, the difference in 5-year overall survival rate was statistically significant $(66 \%$ in the group receiving adjuvant therapy and $55 \%$ in the radiotherapy group; $p=0.03$ ) [12]. These data demonstrate that hormonal treatment in addition to primary therapy, in patients with localized or locally advanced disease, delays the rate of disease progression and can improve overall survival in some patients.

The benefits of adjuvant hormonal therapy in terms of time to disease progression and overall survival were also demonstrated by Granfors et al. [13]. Of 91 patients with T1-4, N0-3, M0 prostate cancer at a median follow-up of 9.3 years, clinical progression was seen in $31 \%$ of patients receiving adjuvant therapy (radiotherapy plus orchiectomy) and $61 \%$ of patients receiving radiotherapy alone ( $\mathrm{p}=$ $0.005)$. Mortality from prostate cancer was $38 \%$ in the adjuvant treatment group, and $61 \%$ in patients receiving radiotherapy alone $(\mathrm{p}=0.02)$. Only patients with lymph node involvement receiving adjuvant therapy had a significant difference in overall survival in comparison with patients receiving radiotherapy alone $(p<0.001)$. No statistically significant survival benefit was identified for patients without lymph node involvement. However, 3 patients receiving adjuvant therapy refused an orchiectomy, although 2 eventually received treatment upon disease progression. Including these 3 patients with those receiving radiotherapy alone, a significant difference for disease-specific survival was seen between the two groups $(p=0.02)$. The high incidence of progression in patients receiving radiotherapy alone resulted in the trial being stopped for ethical reasons before sufficient numbers of patients were recruited for overall analysis. These findings indicate that hormonal therapy adjuvant to radiotherapy may delay disease progression, especially when lymph nodes are involved.

Hanks et al. [14] studied the effects of adjuvant hormonal therapy for 24 months following primary therapy. Patients $(n=1,554)$ with locally advanced disease (T2cT4) received goserelin plus flutamide for 2 months prior to radiotherapy, and were then randomized to no further therapy or to 24 months of additional goserelin alone. After a follow-up of 4.8 years, disease-free survival had increased in the group receiving goserelin therapy compared with those who received no further therapy after radiotherapy (54\% vs. $34 \%$; $=0.0001)$ [14].

Delaying Tumour Progression after Primary Therapy

\section{Adjuvant Hormonal Therapy in the Surgical Setting}

Adjuvant hormonal therapy has also proven to be beneficial in delaying disease progression when used in conjunction with radical prostatectomy. The potential benefit of immediate adjuvant hormonal therapy in a surgical setting was confirmed in a retrospective, non-randomized study by Zincke et al. [15] who examined records for 370 patients with stage $\mathrm{D} 1(\mathrm{~N}+)$ disease who had undergone radical prostatectomy with immediate or delayed adjuvant hormonal therapy. After a median follow-up of 4.1 years, 10-year progression-free survival was significantly higher for those receiving immediate adjuvant therapy compared with those receiving delayed therapy ( $76 \%$ vs. $24 \%$, respectively; $\mathrm{p}<0.0001$ ).

In a randomized clinical trial, Messing et al. [16] demonstrated the effects of adjuvant hormonal therapy (goserelin, $3.6 \mathrm{mg}$ subcutaneously every 28 days or orchiectomy) after radical prostatectomy and pelvic lymphadenectomy in patients with node-positive prostate cancer $(\mathrm{n}=$ 98). After a median follow-up of 7.1 years (range 3-10 years), only $6.4 \%$ of patients in the group receiving adjuvant hormonal therapy died from prostate cancer, compared with $31.4 \%$ of patients receiving deferred hormonal therapy (HR 6.2; 95\% CI 1.8, 21.5; $\mathrm{p}<0.01$ ). Overall survival was also higher in those receiving immediate therapy compared with those receiving deferred therapy (85.2 vs. $64.7 \%$, respectively; HR 3.0; $95 \%$ CI 1.2, 7.3; $\mathrm{p}=0.02$; fig. 2) [16]. However, the results from this trial need to be interpreted with caution because of the small number of patients involved.

In a larger randomized study involving 201 patients with early (T3) prostate cancer who had undergone radical prostatectomy, a $25.2 \%$ benefit in disease-free survival was seen in patients receiving goserelin $(3.6 \mathrm{mg}$ every 28 days) compared with patients who received no treatment after radical prostatectomy [17].

Interim analysis of a further study involving 283 patients with T3, pN0 disease has demonstrated that patients receiving flutamide $250 \mathrm{mg}$ adjuvant to prostatectomy have significantly higher progression-free survival at 4 years compared with those undergoing radical prostatectomy alone (90 vs. $69 \%$, respectively; $\mathrm{p}=0.0029$ ) [18]. Further follow-up is required in this study to establish whether overall survival is significantly improved by adjuvant therapy.

Although hormonal therapy adjuvant to radiotherapy or surgery appears to offer significant increases in time to disease-progression and, in some patients, improved

Eur Urol 2001;40(suppl 2):17-23 
Fig. 2. Overall survival in patients receiving prostatectomy plus adjuvant hormonal therapy (orchiectomy or goserelin) compared with prostatectomy and deferred hormonal treatment [16] (reproduced with kind permission from the Massachusetts Medical Society).

Fig. 3. Progression-free survival after treatment with bicalutamide $150 \mathrm{mg}$ in addition to standard care or standard care alone [22] (reproduced with kind permission from Elsevier Science).
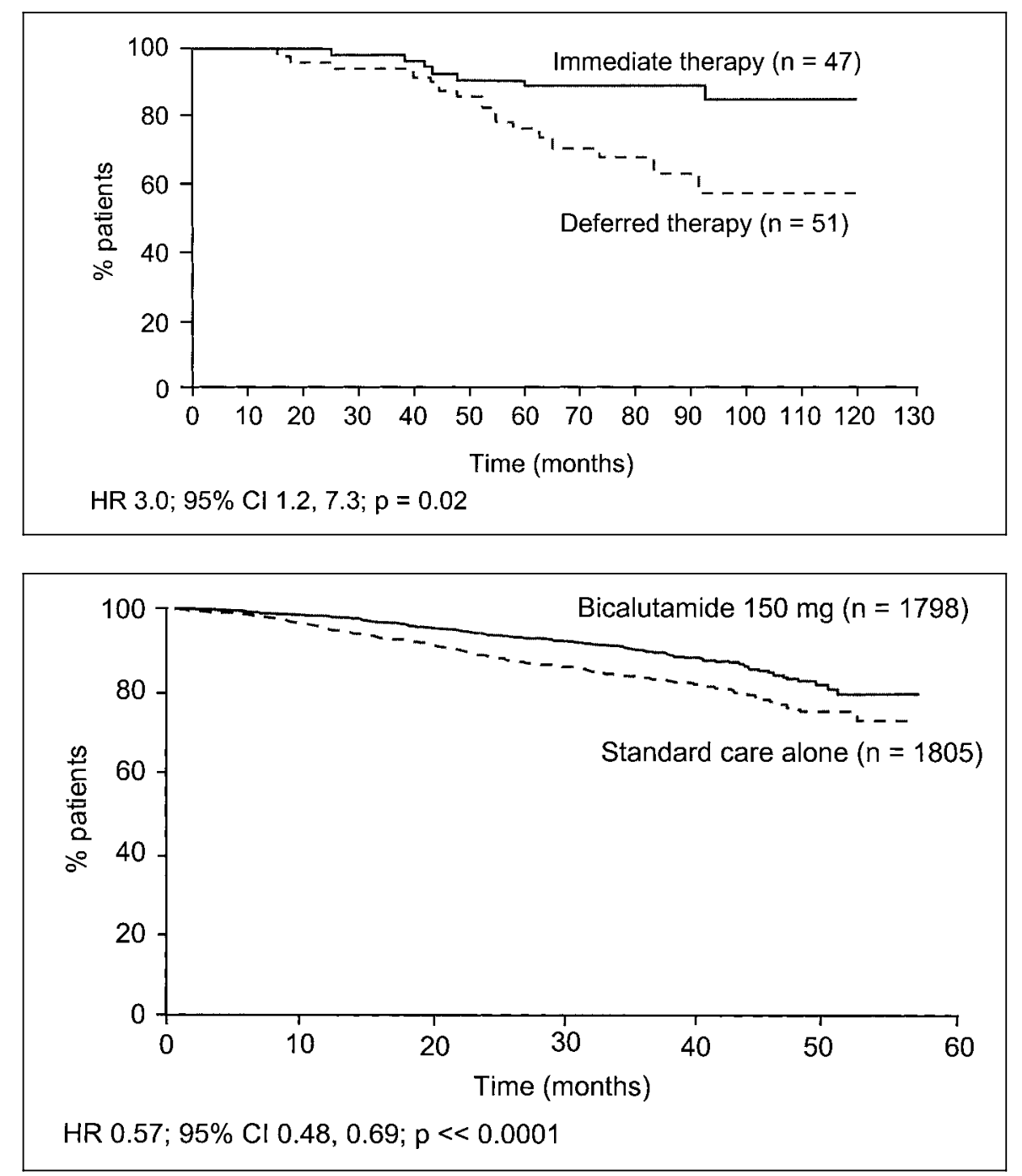

overall survival, there is a need for additional treatments that are effective and well tolerated. The non-steroidal antiandrogen bicalutamide ('Casodex') $150 \mathrm{mg}$ seems to fulfil these requirements and is currently undergoing investigation as immediate therapy, either alone or as adjuvant to standard therapy, in early prostate cancer.

\section{Bicalutamide Therapy in Prostate Cancer}

Bicalutamide $150 \mathrm{mg}$ monotherapy has been shown to provide a survival outcome that is not statistically different to castration in locally advanced, non-metastatic prostate cancer (T3-T4, M0-M1), while offering additional quality of life and tolerability benefits [19]. Bicalutamide does not decrease testosterone concentrations [20], unlike medical or surgical castration, and this may be of particular relevance to patients as sexual potency is less likely to be reduced. Of 480 patients with T3-T4, M0-M1 disease, sexual interest was significantly higher in patients receiving bicalutamide $150 \mathrm{mg}$ than castration $(\mathrm{p}=0.029)$ [19].

The efficacy and tolerability of bicalutamide $150 \mathrm{mg}$ in early prostate cancer as immediate therapy, either alone or as adjuvant therapy, in patients with localized or locally advanced prostate cancer is currently being investigated in the largest $(n=8,113)$ international prostate cancer programme to date [21]. The Early Prostate Cancer (EPC) programme consists of three randomized, doubleblind, placebo-controlled, parallel-group trials. The trials were designed and powered at the outset to support a combined, per protocol analysis. Trial 23 is being carried out in the USA and Canada and has enrolled 3,292 
Table 1. Demography in Trial 24 in the EPC programme [22] (adapted with kind permission from Elsevier Science)

\begin{tabular}{lll}
\hline & $\begin{array}{l}\text { Bicalutamide } 150 \mathrm{mg} \\
(\mathrm{n}=1,798)\end{array}$ & $\begin{array}{l}\text { Standard care alone } \\
(\mathrm{n}=1,805)\end{array}$ \\
\hline Mean age (range), years & $68.6(42-93)$ & $68.7(46-93)$ \\
Preprocedure PSA geometric mean, $\mu \mathrm{g} / \mathrm{l}$ & 12.5 & 11.9 \\
Stage of disease, \% & & \\
$\quad$ T1/T2 & 64.3 & 66.3 \\
T3 & 33.2 & 31.2 \\
T4 & 2.6 & 2.5 \\
Nodal status, \% & 61.3 & 60.4 \\
N0 & 36.0 & 36.9 \\
Nx & 2.6 & 2.7 \\
N+ & & 43.4 \\
Prior therapy, \% & 44.9 & 18.0 \\
Radical prostatectomy & 18.6 & 36.9 \\
Radiotherapy & 34.9 & 1.6 \\
Watchful waiting & 1.6 & \\
Radical prostatectomy plus radiotherapy & &
\end{tabular}

patients, Trial 24 has 3,603 patients recruited from Europe, Australia, South Africa, Israel and Mexico, and Trial 25 has 1,218 patients in Denmark, Norway, Finland and Sweden.

Primary endpoint data for time to progression are now available for Trial 24 [22]. Patients with stage T1b-T4, any nodal status, M0 prostate cancer were randomised to receive bicalutamide $150 \mathrm{mg}$ or placebo once daily (oral dose). Patients had received no previous therapy (35.9\%), radical prostatectomy $(44.2 \%)$, radiotherapy $(18.3 \%)$ or radical prostatectomy plus radiotherapy (1.6\%). The treatment groups were well matched for demography, tumour stage and grade, nodal status and prior therapy (table 1) [22].

Bicalutamide $150 \mathrm{mg}$ or placebo was administered once daily, and the recommended duration of treatment was 5 years when given as adjuvant therapy or until disease progression in patients undergoing watchful waiting. Of the 3,603 patients recruited to the study, 1,798 received bicalutamide $150 \mathrm{mg}$ and 1,805 received standard care alone. Objective disease progression was determined by a positive bone scan, or other bio-imaging techniques e.g. computed tomography, magnetic resonance imaging or ultrasound scan. Changes in PSA or clinical examination findings alone were not considered to be evidence of objective progression.

After a median follow-up of 2.6 years, the risk of objective progression of disease was significantly reduced (by $43 \%$ ) in patients receiving bicalutamide $150 \mathrm{mg}$ compared with those receiving standard care alone (HR 0.57; 95\% CI 0.48, 0.69; $\mathrm{p}<<0.0001$; fig. 3) [22].

Delaying Tumour Progression after Primary Therapy

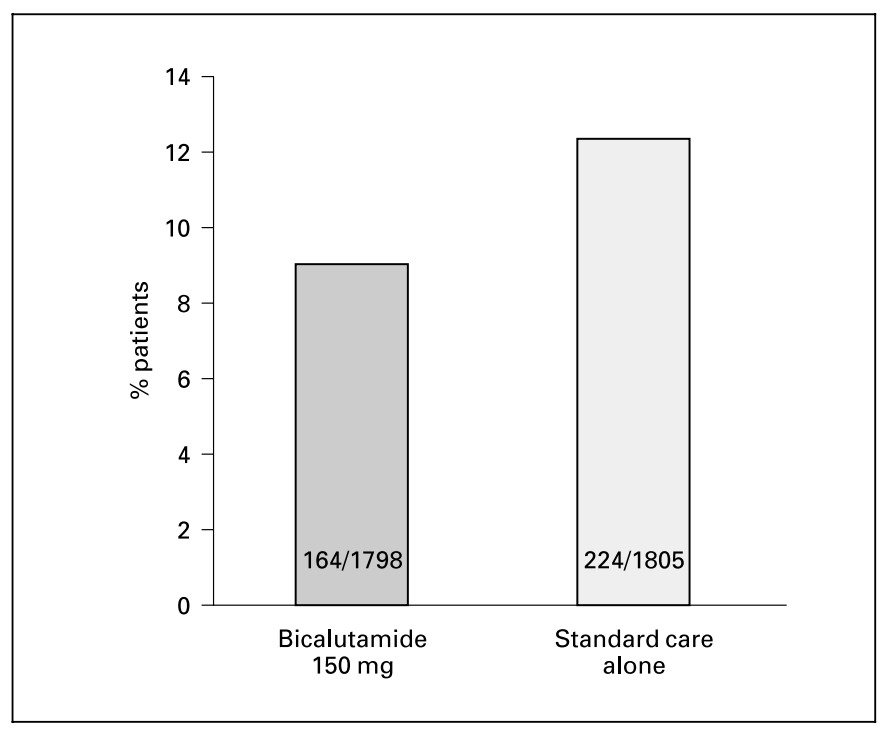

Fig. 4. Incidence of bone scan confirmed progression after treatment with bicalutamide $150 \mathrm{mg}$ in addition to standard care or standard care alone.

The incidence of bone scan-confirmed progression or death indicates development of metastatic disease. Development of metastatic disease is an important prognostic factor for patients with prostate cancer, as survival is decreased in patients with metastatic disease [23]. The incidence of positive bone scans was reduced in the bicalutamide $150 \mathrm{mg}$ group $(9.1 \%)$ compared with the standard care alone group (12.4\%; fig. 4).

The benefits of bicalutamide $150 \mathrm{mg}$ were seen across the patient population studied, regardless of standard

Eur Urol 2001;40(suppl 2):17-23 


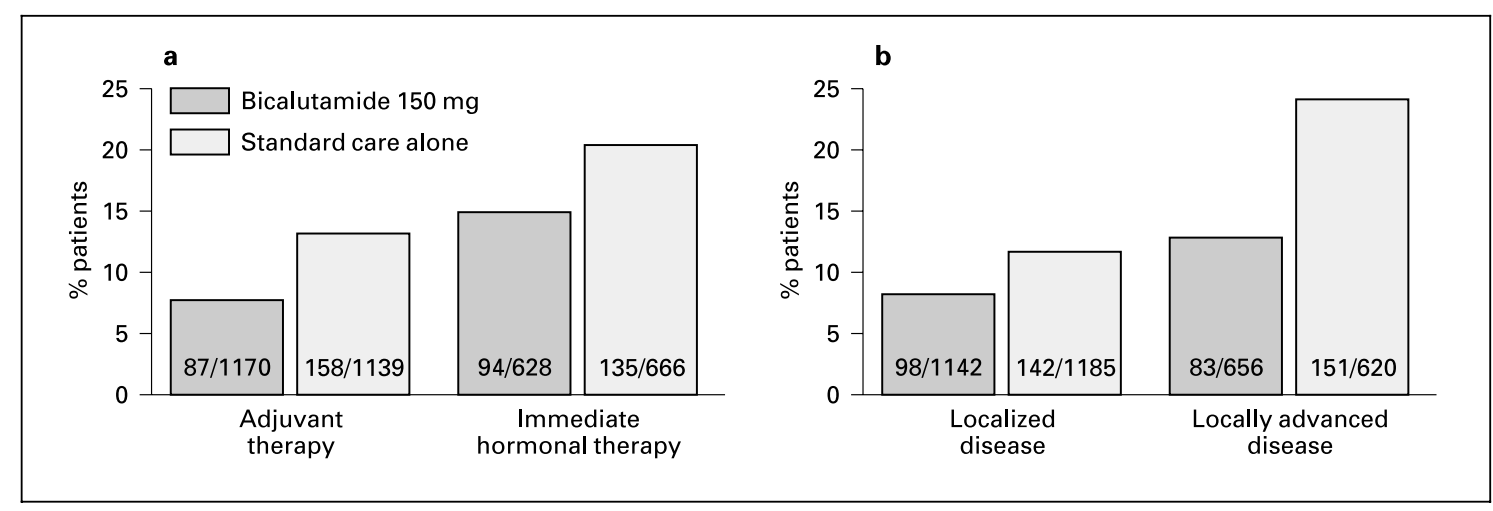

Fig. 5. Incidence of objective progression after treatment with bicalutamide $150 \mathrm{mg}$ in addition to standard care or standard care alone classified by (a) prior therapy and (b) stage of disease [22] (reproduced with kind permission from Elsevier Science).

Table 2. Adverse events in Trial 24 in the EPC programme [22] (adapted with kind permission from Elsevier Science)

\begin{tabular}{lll}
\hline & $\begin{array}{l}\text { Bicalutamide } \\
150 \mathrm{mg}, \% \\
(\mathrm{n}=1,798)\end{array}$ & $\begin{array}{l}\text { Standard care } \\
\text { alone, \% } \\
(\mathrm{n}=1,805)\end{array}$ \\
\hline Gynaecomastia alone & 17.4 & 5.3 \\
Breast pain alone & 17.6 & 3.1 \\
Gynaecomastia plus breast pain & 47.5 & 2.1 \\
Hot flushes & 9.3 & 4.6 \\
Impotence & 8.0 & 5.3 \\
Asthenia & 7.2 & 6.1 \\
Weight gain & 5.6 & 2.6 \\
Diarrhoea & 5.1 & 6.3 \\
Loss of libido & 2.6 & 0.6 \\
Elevated liver enzymes & 2.7 & 0.6 \\
\hline
\end{tabular}

therapy or disease stage. In patients who had previously undergone therapy of curative intent, a lower incidence of objective progression was observed in patients receiving bicalutamide $150 \mathrm{mg}$ than in those receiving standard care alone ( 7.4 vs. $13.9 \%$, respectively). In patients who were candidates for watchful waiting, the incidence of objective progression was also lower in patients who received bicalutamide $150 \mathrm{mg}$ compared with those who received standard care alone (15.0 vs. $20.3 \%$, respectively). In patients with localized disease, fewer of those receiving bicalutamide $150 \mathrm{mg}$ progressed than those receiving standard care alone (8.6 vs. $12.0 \%$, respectively). In patients with locally advanced disease a larger difference was apparent (12.7 vs. $24.4 \%$, respectively; fig. 5) [22].
These results were supported by data on PSA progression. PSA progression was defined as at least a doubling in PSA from baseline ( $91 \%$ of patients receiving radical prostatectomy had a PSA concentration of $<1 \mathrm{ng} / \mathrm{ml}$ ), or any objective clinical progression or death (i.e. biochemical and clinical progression-free survival). Risk of PSA progression was significantly reduced (by 63\%) in the bicalutamide $150 \mathrm{mg}$ group, with only $15 \%$ of patients receiving bicalutamide $150 \mathrm{mg}$ experiencing PSA progression at the time of analysis compared with $33 \%$ of patients receiving standard care alone (HR $0.37 ; 95 \%$ CI $0.32,0.43 ; \mathrm{p}<<0.0001)$. Only $260(7.2 \%)$ of the patients (123 receiving bicalutamide $150 \mathrm{mg}$ vs. 137 receiving standard care alone) have died at the time of this first analysis, thus preventing a formal statistical analysis, and therefore longer follow-up is needed to provide meaningful data on survival.

The tolerability profile of bicalutamide $150 \mathrm{mg}$ was as would be expected from its pharmacology. Gynaecomastia and breast pain were the most frequent side effects in the bicalutamide $150 \mathrm{mg}$ group, but the incidence of hot flushes, impotence and loss of libido were relatively low (table 2) [22]. There was a low incidence of abnormal liver function tests (2.7 vs. $0.6 \%)$ and rises were frequently transient and rarely severe.

Withdrawal rates were similar in the bicalutamide $150 \mathrm{mg}$ and standard care alone groups (40.3 vs. $37.2 \%$, respectively). Withdrawals due to gynaecomastia and breast pain were $15.4 \%$ in patients receiving bicalutamide $150 \mathrm{mg}$, compared with $0.5 \%$ in patients receiving standard care alone. More patients in the bicalutamide $150 \mathrm{mg}$ group withdrew due to side effects compared with the standard care alone group ( 24.5 vs. $7.7 \%$, respective- 
ly), but fewer patients in the bicalutamide $150 \mathrm{mg}$ group withdrew due to objectively confirmed disease progression than in the standard care alone group (2.8 vs. $10.8 \%$, respectively).

These first results from the EPC programme show that bicalutamide $150 \mathrm{mg}$ as an immediate therapy, either alone or as adjuvant to primary therapy, reduces disease progression for most patients, with acceptable side effects. Therefore, bicalutamide $150 \mathrm{mg}$ may be a suitable primary or adjuvant therapy for the long-term treatment of patients with localized or locally advanced prostate cancer who are at risk of tumour progression. As early diagnosis of prostate cancer improves, increasing numbers of patients with localized disease are likely to be identified and these patients will require an effective and well-tolerated treatment regimen.

\section{Conclusion}

Adjuvant hormonal therapies offer an extended period of progression-free survival and may improve overall survival in some patients.

The first results from a study in the EPC programme, the largest ever international prostate cancer programme to date, show a statistically significant benefit for bicalutamide $150 \mathrm{mg}$ in reducing the risk of objective progression when given as immediate therapy either alone or as adjuvant to treatment of curative intent in patients with localized or locally advanced prostate cancer. Survival data are eagerly awaited.

\section{References}

1 Landis SH, Murray T, Bolden S, Wingo PA: Cancer statistics, 1999. CA Cancer J Clin 1999; 49:8-31.

2 Hall RR: Screening and early detection of prostate cancer will decrease morbidity and mortality from prostate cancer: The argument against. Eur Urol 1996;29(suppl 2):24-26.

3 McPherson CP, Swenson KK, Kjellberg J: Quality of life in patients with prostate cancer. Semin Oncol Nurs 2001;17:138-146.

4 Epstein JI, Partin AW, Sauvageot J, Walsh PC: Prediction of progression following radical prostatectomy. A multivariate analysis of 721 men with long-term follow-up. Am J Surg Pathol 1996;20:286-292.

5 Roach M, Lu J, Pilepich MV, Asbell SO, Mohiuddin M, Terry R, Grignon D, Mohuidden M: Four prognostic groups predict long-term survival from prostate cancer following radiotherapy alone on Radiation Therapy Oncology Group clinical trials. Int J Radiat Oncol Biol Phys 2000;47:609-615.

6 Townsend MF, Sanders WH, Northway RO, Graham SD Jr: Bone fractures associated with luteinizing hormone-releasing hormone agonists used in the treatment of prostate carcinoma. Cancer 1997;79:545-550.

7 Weber JP, Walsh PC, Peters CA, Spivak JL: Effect of reversible androgen deprivation on hemoglobin and serum immunoreactive erythropoietin in men. Am J Hematol 1991;36:190_ 194.

8 Dreicer R: The evolving role of hormone therapy in advanced prostate cancer. Cleve Clin $\mathrm{J}$ Med 2000;67:720-722, 725-726.

9 Isaacs JT: The timing of androgen ablation therapy and/or chemotherapy in the treatment of prostatic cancer. Prostate 1984;5:1-17.

10 Isaacs JT: Relationship between tumor size and curability of prostatic cancer by combined chemo-hormonal therapy in rats. Cancer Res 1989;49:6290-6294.
11 Bolla M, Gonzalez D, Warde P, Dubois JB, Mirimanoff RO, Storme G, Bernier J, Kuten A, Sternberg C, Gil T, Collette L, Pierart M: Improved survival in patients with locally advanced prostate cancer treated with radiotherapy and goserelin. N Engl J Med 1997;337:295300.

12 Pilepich MV, Caplan R, Byhardt RW, Lawton CA, Gallagher MJ, Mesic JB, Hanks GE, Coughlin CT, Porter A, Shipley WU, Grignon D: Phase III trial of androgen suppression using goserelin in unfavorable-prognosis carcinoma of the prostate treated with definitive radiotherapy: Report of Radiation Therapy Oncology Group Protocol 85-31. J Clin Oncol 1997; 15:1013-1021.

13 Granfors T, Modig H, Damber JE, Tomic R: Combined orchiectomy and external radiotherapy versus radiotherapy alone for nonmetastatic prostate cancer with or without pelvic lymph node involvement: A prospective randomized study. J Urol 1998;159:2030-2034.

14 Hanks GE, Lu J, Machtay M, Venkatesan V, Pinover W, Byhardt R, Rosenthal SA: RTOG Protocol 92-02: A Phase III trial of the use of long term androgen suppression following neoadjuvant hormonal cytoreduction and radiotherapy in locally advanced carcinoma of the prostate. Proc ASCO 2000:Abstract 1284.

15 Zincke H, Bergstralh EJ, Larson-Keller JJ, Farrow GM, Myers RP, Lieber MM, Barrett DM, Rife CC, Gonchoroff NJ: Stage D1 prostate cancer treated by radical prostatectomy and adjuvant hormonal treatment. Evidence for favorable survival in patients with DNA diploid tumors. Cancer 1992;70(suppl 1):311-323.

16 Messing EM, Manola J, Sarosdy M, Wilding G, Crawford ED, Trump D: Immediate hormonal therapy compared with observation after radical prostatectomy and pelvic lymphadenectomy in men with node-positive prostate cancer. N Engl J Med 1999;341:1781-1788.
17 Prayer-Galetti T, Zattoni F, Capizzi A, Dal Moro F, Pagano F: Study group disease free survival in patients with pathological ' $\mathrm{C}$ stage' prostate cancer at radical retropubic prostatectomy submitted to adjuvant hormonal treatment. Eur Urol 2000;38:504 (Abstract 48).

18 Wirth M, Froehner M: A review of studies of hormonal adjuvant therapy in prostate cancer. Eur Urol 1999;36(suppl 2):14-19.

19 Iversen P, Tyrrell CJ, Kaisary AV, Anderson JB, Van Poppel H, Tammela TL, Chamberlain M, Carroll K, Melezinek I: Bicalutamide monotherapy compared with castration in patients with nonmetastatic locally advanced prostate cancer: 6.3 years of followup. J Urol 2000;164:1579-1582.

20 Verhelst J, Denis L, Van Vliet P, Van Poppel H, Braeckman J, Van Cangh P, Mattelaer J, D'Hulster D, Mahler C: Endocrine profiles during administration of the new non-steroidal anti-androgen Casodex in prostate cancer. Clin Endocrinol (Oxf) 1994;41:525-530.

21 See WA, McLeod D, Iversen P, Wirth M: The bicalutamide Early Prostate Cancer Program: Demography. Urol Oncol 2001;6:43-47.

22 Wirth M, Tyrrell C, Wallace M, Delaere KP, Sanchez-Chapado M, Ramon J, Hetherington J, Pina F, Heynes CF, Borchers TM, Morris T, Stone A: Bicalutamide ('Casodex') $150 \mathrm{mg}$ as immediate therapy in patients with localized or locally advanced prostate cancer significantly reduces the risk of disease progression. Urology 2001;58:146-151.

23 Liebel SA, Fuks Z, Zelefsky MJ, Whitmore WF Jr: The effects of local and regional treatment on the metastatic outcome in prostatic carcinoma with pelvic lymph node involvement. Int $\mathbf{J}$ Radiat Oncol Biol Phys 1994;28:7-16. 